\title{
Rotational Invariance, the Spin-Statistics Connection and the TCP Theorem
}

\author{
E.C.G. Sudarshan \\ University of Texas, Austin, TX,USA
}

\begin{abstract}
Quantum Field Theory formulated in terms of hermitian fields automatically leads to a spin-statistics connection when invariance under rotations is required. In three (or more) dimensions of space this implies Bose statistics for integer spin fields and Fermi statistics for half-integer spin fields. One should recall that spin- $1 / 2$ fields in three dimensions have two nonhermitian or four hermitian components. This automatic doubling of the number of components enables one to define a pseudoscalar matrix, and this in turn allows one to prove the TCP theorem for rotationally invariant field theories. In two space dimensions one obtains anyon statistics independent of the "spin". For the quantum mechanics of identical particles we obtain only the possibility of either statistics for either spin as long as the spatial dimension is three (or higher). For two space dimensions we get anyon statistics. This difference is due to the contractibility of closed loops in three or more dimensions. The relation to the arguments of Broyles, of Bacry and of Berry and Robbins is discussed.
\end{abstract}

\section{INTRODUCTION}

In the description of dynamics of identical particles in either quantum theory or classical theory the Hamiltonian is symmetric under any permutation of the particles; therefore, if the initial conditions have any definite symmetry, it would be preserved by the dynamics. It therefore follows that the problem of symmetries of the dynamics is purely a kinematics problem. Since in classical physics the state is usually described by a phase space density, which evolves linearly in time, the only choice, is to use symmetric kinematics. This may also be seen as the vestige of Bose statistics for integer spin particles. If we transcribe quantum dynamics in terms of Moyal brackets in phase space and keep only the lowest order terms in Planck's constant we will have a classical Poisson bracket formulation for classical fields which have integer spin. If we use more sophisticated classical dynamics and kinematics in terms of spinor variables, like in the Proca description of ideal fluid dynamics, we could legitimately raise the possibility of anticommuting spinor 'urfields'. Ideal fluid dynamics per se can be given a canonical formulation in terms of Clebsch potentials. In classical particle mechanics we always deal with integer spin particles and phase space densities which must, of necessity, be totally symmetric. If there are substructures, which yield the particles as bilinear composites, we could entertain 
more general possibilities. In passing we recognize that the question of the kinematic symmetries for field theories is distinct from the corresponding question for particles.

It is natural to ask if there is a continuous path connecting a configuration of particles with another configuration of particles with some of the particles permuted, would this not settle the problem of statistics? Several attempts have been made to date. The simplest is by $\mathrm{H}$. Bacry [1] who took a configuration of two spin-1/2 particles polarized, perpendicular to their separation, and in opposite directions. He then showed that if we carry out a rotation through $\pi$ of the system of two particles about the center of their separation, we get a configuration identical with the original configuration. So if we conclude that the wave function returns to its value at the end of this rotation, then we can deduce Fermi statistics for electrons. But there is a hidden assumption. If we chose commuting Bose operators on the vacuum to create the two electrons, such a path will change the wave function by a factor of -1 . In the space of configurations the beginning and ending configurations are identical; so we have a closed loop. But not all closed loops automatically preserve the wave function. It will be preserved if the "loop" can be continually contracted to the identity. But the loop considered by Bacry is not so contractible. In general, if we have a closed loop in the space of configurations there is no guarantee that the wave function is preserved unless the configuration space is simply connected [2].

This question has been studied in its full generality by T.D. Imbo and collaborators [3]. If we consider a base manifold $M$, which may be $\mathrm{R}^{3}$ or it could itself be multiply connected, and we have $N$ identical particles, the configurations in which two particles are exchanged are not different. So the possible manifold is

$$
\frac{M \otimes M \otimes \ldots \otimes M}{S_{N}}=\frac{M^{N}}{S_{N}},
$$

where $S_{N}$ is the permutation group on $N$ variables. But this is not a differentiable manifold unless we remove $\Delta_{N}$, the set of points where two or more coordinates coincide. We do so. The appropriate manifold is then

$$
S=\frac{M^{N}-\Delta_{N}}{S_{N}} .
$$

This is a differentiable manifold but it is multiply connected. The connectivity is summarized in its loop group, the first homology group $\pi_{1}(S)$, which may be nontrivial. If $M$ is simple connected, we have

$$
\pi_{\mathrm{l}}(S)=\pi_{1}\left(\frac{M^{N}-\Delta_{N}}{S_{N}}\right)=S_{N},
$$

provided we work in three or more dimensions. The generic wave functions are multi-valued, formalizing a representation of $S_{N}$. Apart from the completely symmetric and completely antisymmetric representations, there are no further onedimensional representations. Since we wish to have one-dimensional representations, only the Bose and Fermi statistics obtain. Of course, all the parastatistics are also 
allowed if we relax the requirement of only one-dimensional representations. For dimension one, the space $S$ is not even connected. For dimension two, the homotopy group is the braid group

$$
B_{N}(M)=\pi_{1}(S)=\pi_{1}\left(\frac{M^{N}-\Delta_{N}}{S_{N}}\right)
$$

There is a continuous family of one-dimensional representations of the braid group labeled by a phase $0 \leq \theta \leq 2 \pi$. These particles are called anyons.

Note that there is no correlation between the representations of the connectivity group $\pi_{1}(S)$ and the spin of the particles.

The argument of Bacry is equivalent to disregarding all except the trivial identity representation of the homotopy group. The existence of a continuous path following a loop gives no guarantee of the representation being trivial. If one asserts that the wavefunction remains unchanged, it is an additional explicit assumption [1,2].

\section{REQUIREMENTS FOR A QUANTUM FIELD THEORY}

In the quantum mechanics of particles, we have a description in terms of wave functions (or state vectors), which enter bilinearly in the computation of expectation values. Therefore a completely antisymmetric as well as completely symmetric wave function gives us completely symmetric expectation values. Even if the permutation of identical particles gives a phase change, not necessarily \pm 1 , it will cancel out in the rule for taking expectation values. Since wave functions allow for superposition of any two states to form a new state, all the wavefunctions of a collection of identical particles must have the same behavior. We may then deal with the symmetry properties of a collection of identical particles without special reference to any state or collection of states.

For particles that can be separated from each other, we need to have some restrictions on the symmetry due to the requirement of "cluster decomposition" [4]. If a set of $M+N$ particles has a wave function with a definite symmetry property, it should be such that when the cluster of $M$ particles is arbitrarily far from the cluster of $N$ particles each cluster has the "same" symmetry property. To require this is to assume that the clusters can be separated arbitrarily far: this property does not obtain for "particles" such as quarks which are "confined".

In quantum theory the permutations of the particles form a group of operations which will have a set of possible representations. In this manner, finding the appropriate "statistics" for a particle species can be reduced to finding the representations of the groups that interchange configurations [3].

Within the framework of relativistic quantum field theory, W.Pauli [5] demonstrated a spin-statistics relation. He used Lagrangian field theory with scalar or spinor wavefields. This well known and celebrated result asserts that scalar (or 
pseudoscalar) fields cannot be consistently quantized in accordance with Fermi statistics; similarly, spinor fields cannot be consistently quantized in accordance with Bose statistics. Thus the theorem is of wide applicability provided we deal only with Bose or Fermi quantizations. Since there are other possibilities, we need to reexamine this question. Pauli's method of proof suffers from the defect that it applies directly for free fields only. When interactions are included, it is a hoped that these same conclusions obtain. Since the statistics is a kinematics question (since dynamics preserves the symmetry of the initial conditions), a proper formulation can eliminate this objection.

But the main reasons why we should search for a formulation not invoking relativistic invariance are two-fold. For one, all the places where the spin-statistics relation is most important like atomic and molecular structure, blackbody radiation, and condensed matter physics, the velocities of the electrons are sufficiently small so that relativistic effects can be neglected. For the second, we have quantized sound waves with phonons, the quantum electrodynamics of wave-guides, and the excitation spectrum of an ideal fluid. So it would be desirable to find a proof of the spinstatistics relation without directly invoking relativistic invariance or particle statistics with renormalized fields. It is nevertheless taken for granted that the interactions do not affect the statistics. However, in $2+1$ dimensions the special theory with a ChernSimons kinematic term [5] in interaction with a charge-current transmutes its statistics, with the low frequency components obeying one statistics and the high frequency components obeying another statistics [6]. The 2+1-dimensional theory is special in that in addition to Bose and Fermi statistics, a one-parameter family of anyon statistics may obtain. But it does caution us in asserting that the free-field commutation relations imply the interacting-particle statistics.

\section{FIELD STATISTICS WITHOUT PARTICLES}

A direct study of the field commutation relations without specific reference to the interaction is provided by axiomatic quantum field theory. Such a formulation was provided by A.S. Wightman. The Wightman scheme defines the quantized fields as operator-valued distributions acting on a Hilbert space. The fields admit a family of automorphisms corresponding to different Poincaré frames, and these are unitarily implemented. The energy is always nonnegative and there is a unique vacuum state [8], which is left invariant by the family of Poincaré automorphisms. No specific form of interactions is postulated, or required. Within this scheme, N. Burgoyne [9] has given a proof of the connection between the spin of the field (tensor or spinor) and its commutation properties. The proof goes as follows. Consider the vacuum expectation values of the product of two field operators at two space-time points that are separated by a space like interval. From Poincaré invariance we deduce 


$$
\langle 0|\phi(x) \phi(y)| 0\rangle=F(x-y)
$$

where $F$ is a Lorentz-invariant function of the separation. They must then be functions of the Lorentz-invariant quantities that can be constructed from the interval. For time-like intervals both $(x-y)^{2}=(\vec{x}-\vec{y})^{2}-c^{2}\left(x^{0}-y^{0}\right)^{2}$ and $\theta\left(x^{\circ}-y^{\circ}\right)^{2}$ are invariants, the first symmetric and the second antisymmetric in $x$ and $y$. But for space-like intervals, only the symmetric invariant $(x-y)^{2}$ obtains. It follows that

$$
\langle 0|\phi(x) \phi(y)| 0\rangle=\langle 0|\phi(y) \phi(x)| 0\rangle, \quad x-y \text { spacelike , }
$$

and hence the commutator has zero vacuum expectation value. If we now demand that the field is quantized with anticommutators,

$$
\phi(x) \phi(y)+\phi(y) \phi(x)=0, \quad c^{2}\left(x^{0}-y^{0}\right)^{2}<(\vec{x}-\vec{y})^{2},
$$

it will follow that

$$
\langle 0|\phi(x) \phi(y)| 0\rangle=0 \text {. }
$$

This implies that

$$
\phi(x)|0\rangle=0
$$

and that the field is trivially zero. For spinor fields a similar analysis leads to the conclusion that

$$
\left\langle 0\left|\psi_{r}(x) \psi_{s}(y)\right| 0\right\rangle=-\left\langle 0\left|\psi_{s}(x) \psi_{r}(y)\right| 0\right\rangle
$$

for $x-y$ space-like. Consequently, imposition of the requirement that the commutators of two fields with space-like separation vanish gives

$$
\left\langle 0\left|\psi_{r}(x) \psi_{s}(y)\right| 0\right\rangle=0 \quad \psi_{r}(y)|0\rangle=0,
$$

so the field is again trivial. We note that the Burgoyne proof is also a negative result: integer spin fields cannot obey Fermi statistics and half-integer spin fields cannot obey Bose statistics. But the field has no immediate particle interpretation; if there were, then the negative proof may be applied to the corresponding particles.

Similar results are also obtained within the framework of the $\mathrm{C}^{*}$ algebra formulation [10] of D.Kastler and R.Haag. Again there is no immediate particle interpretation, and the theorems are negative results.

We can take the lessons of both Pauli's proof and Burgoyne's and ask if we can deduce these negative results from weaker assumptions, especially releasing relativistic invariance. We can further ask if we can deduce a positive result that the tensor fields must satisfy Bose statistics and that the spinor fields must satisfy Fermi statistics. This can be accomplished in a suitably restricted Lagrangian field theory invariant under the Newtonian group $N(3,1)$ comprising space and time translations and space rotations. There must be some restrictions, since we could consider nonrelativistic quantum field theories with either statistics for both tensor and spinor fields.

Before giving the requirements on the $\mathrm{N}(3,1)$ invariant fields, we need to answer a question raised by internal symmetries. What if we do have internal symmetry multiplets of tensor (spinor) fields which overcome the limitations imposed by 
Burgoyne, by making the vacuum expectation values antisymmetric in the internal symmetry variables? Does this reverse the conclusions? A careful analysis eliminates this possibility. Any internal symmetry dependence can be diagonalized by suitable global linear combinations. An antisymmetric matrix would have pure imaginary eigenvalues of either sign. The action density is then pure imaginary and of either sign. Such a field theory is not acceptable. So we cannot evade the Burgoyne constraints by invoking antisymmetry in internal symmetry labels.

\section{EXOTIC STATISTICS}

It is interesting and useful to ask what can be asserted about particle statistics without relativity and without fields [9]. In this case we specify the configuration space of $N$ particles as

$$
S=\frac{\mathrm{R}^{3} \times \mathrm{R}^{3} \times \ldots \times \mathrm{R}^{3}-\Delta}{S_{N}},
$$

where $\Delta$ is the set of points in which two or more sets of coordinates coincide and $S_{N}$ is the symmetric group in $N$ variables permuting the different $\mathrm{R}^{3}$ spaces. This space (with $\Delta$ removed) is a manifold on which the wavefunctions can be described.

We can define a set of wavefunctions that transform among themselves when we consider a non-contractible loop in $S$. It is possible for these wave functions to furnish a representation of $S_{N}$. But except for the completely symmetric and completely antisymmetric representations, all the others are nontrivial "parastatistics" [10].

This set of possible statistics emerges from the work of E.P. Wigner and of H.S. Green [11]. They observed that the equations of motion of an assembly of particles realized by the Fock states of a collection of creation and annihilation operators $a_{j}, a_{j}^{\dagger}$ are those of harmonic oscillators

$$
\begin{gathered}
i \dot{a}_{j}=\left[a_{j}, H\right], \\
H=1 / 2 \sum \omega_{k}\left(a_{k}^{\dagger} a_{k} \pm a_{k} a_{k}^{\dagger}\right),
\end{gathered}
$$

so that

$$
i \dot{a}_{j}=\omega_{j} a_{j} \quad i \dot{a}_{j}^{\dagger}=-\omega_{j} a_{j}^{\dagger}
$$

But these do not require the canonical relation

$$
a_{j} a_{k}^{\dagger} \pm a_{k}^{\dagger} a_{j}=\delta_{j k}
$$

It suffices to have

$$
1 / 2\left[\sum \omega_{k}\left(a_{k}^{\dagger} a_{k} \pm a_{k} a_{k}^{\dagger}\right), a_{l}\right]=i \dot{a}_{l} .
$$

Since this must be true independent of the way the orthonormal modes $j, k, \ldots$ are chosen, it follows that we need the generalized commutation relation 


$$
\begin{aligned}
& {\left[a_{j} a_{k}^{\dagger} \pm a_{k}^{\dagger} a_{j}, a_{l}\right]=2 \delta_{k l} a_{j}} \\
& {\left[a_{j} a_{k}^{\dagger} \pm a_{k}^{\dagger} a_{j}, a_{l}^{\dagger}\right]=2 \delta_{k l} a_{j}^{\dagger} .}
\end{aligned}
$$

We may supplement these with

$$
\begin{aligned}
& {\left[a_{j} a_{k} \pm a_{k} a_{j}, a_{l}\right]=0} \\
& {\left[a_{j} a_{k} \pm a_{k} a_{j}, a_{l}^{\dagger}\right]=2\left(\delta_{k l} a_{j} \pm \delta_{j l} a_{k}\right)}
\end{aligned}
$$

and its adjoint. These were the generalized statistics that H.S. Green studied. Wigner had raised the question for one degree of freedom for the harmonic oscillator. H.S. Green gave a generic construction of operators which obey these commutation relations. We shall call this the "Green ansatz" and discuss it below.

But before discussing the Green ansatz let us study the generalized Green commutation relations. First let us take para-Fermi statistics with the commutation relations

$$
\begin{aligned}
& {\left[a_{j}^{\dagger} a_{k}-a_{k} a_{j}^{\dagger}, a_{l}\right]=-2 \delta_{j l} a_{k}} \\
& {\left[a_{j}^{\dagger} a_{k}-a_{k} a_{j}^{\dagger}, a_{l}^{\dagger}\right]=2 \delta_{k l} a_{k}^{\dagger} .}
\end{aligned}
$$

We can use them in this form to find all representations of these commutation relations. But it will be more transparent to label the various degrees of freedom from 1 to $N$ and introduce

$$
\begin{gathered}
J_{1,2 N+1}=1 / 2\left[a_{1}+a_{1}^{\dagger}\right] \quad J_{2,2 N+1}=i 1 / 2\left[a_{1}-a_{1}^{\dagger}\right] \\
\ldots \\
J_{2 N+1,2 N+1}=1 / 2\left[a_{N}+a_{N}^{\dagger}\right] \quad J_{2 N+2,2 N+1}=i 1 / 2\left[a_{N}-a_{N}^{\dagger}\right] \\
J_{1,3}=1 / 4\left[a_{1}+a_{1}^{\dagger}, a_{2}+a_{2}^{\dagger}\right] \quad J_{1,2}=i 1 / 4\left[a_{1}+a_{1}^{\dagger}, a_{2}-a_{2}^{\dagger}\right] \\
\ldots \\
J_{2 N-1,2 N}=i 1 / 4\left[a_{N}+a_{N}^{\dagger}, a_{N}-a_{N}^{\dagger}\right] \quad J_{2 N-2,2 N}=1 / 4\left[a_{N-1}+a_{N-1}^{\dagger}, a_{N}-a_{N}^{\dagger}\right] .
\end{gathered}
$$

Then $J_{\mu v}$, for $1 \leq \mu, \nu \leq 2 N+1$, obey the commutation relation of the orthogonal group $B_{N}=\mathrm{O}(2 N+1)$ in odd dimensions [12]. Therefore the representations we need are all the representation of $B_{N}$, reducible or irreducible. This is a generalization of a result of H.J. Bhabha [13]. All the representation of $B_{N}$ are known; the irreducible ones are listed by F.D. Murnaghan. In particular the standard Fermi realization of this algebra is the fundamental irreducible $2^{N}$ dimensional spinor. 


\section{THE GREEN ANSATZ}

The Green ansatz for this case is to take $R$ families of mutually commuting Fermi operators

$$
\left[b_{j}^{(r)}, b_{k}^{\dagger(s)}\right]=0 ; \quad\left[b_{j}^{(r)}, b_{k}^{(s)}\right]=\left[b_{j}^{\dagger(r)}, b_{k}^{\dagger(s)}\right]=0, \quad j \neq k \quad r \neq s .
$$

Then choose

$$
a_{j}=\Sigma b_{j}^{(r)} \quad a_{j}^{\dagger}=\Sigma b_{j}^{\dagger(r)} .
$$

This ansatz solves Green's commutation relations. It simply corresponds to the vector additions of the spins of $R$ independent spinors. All irreducible representations of $B_{N}$ can obtained by the reduction of this $R$-fold product of spinors of $B_{N}$. Except in the trivial case of $R=1$, the Green ansatz is a reducible realization. The labels $(r, s)$ are inert and are the color labels, and the unitary group in $R$ variables is the color symmetry. We may restrict the representation of color symmetry arbitrarily to the symmetric identity representation. Then our physics would be colorless.

For the para-Bose case the identification is somewhat more complicated. Consider the commutation relation of the bilinear forms $a_{j} a_{k}+a_{k} a_{j}, a_{j} a_{k}^{\dagger}+a_{k}^{\dagger} a_{j}$, and $a_{j}^{\dagger} a_{k}^{\dagger}+a_{k}^{\dagger} a_{j}^{\dagger}$. They satisfy the commutation relations of $C_{N}=S_{p}(2 N)$, the symmetric group in $N$ pairs of variables [14]. The operators $a_{j}, a_{j}^{\dagger}$ may be considered as grading the $C_{N}$. And every representation of this graded Lie algebra consists of a pair of representations of $C_{N}$. These representations are also given be Murnaghan [15].

The Green ansatz for para-Bose commutation relations is to take $R$ mutually anticommuting Bose algebras

$$
\begin{aligned}
& {\left[b_{j}^{(r)}, b_{k}^{(r)}\right]=0 ; \quad\left[b_{j}^{(r)}, b_{k}^{\dagger(r)}\right]=\delta_{j k}} \\
& b_{j}^{(r)} b_{k}^{(s)}=-b_{k}^{(s)} b_{j}^{(r)} \\
& b_{j}^{(r)} b_{k}^{\dagger(s)}=-b_{k}^{\dagger(s)} b_{j}^{(r)} \quad r \neq s, j \neq k
\end{aligned}
$$

and construct

$$
a_{j}=\sum b_{j}^{(r)} \quad a_{j}^{\dagger}=\sum b_{j}^{\dagger(r)} .
$$

Again we get realizations of these para-Bose commutation relations. All these realizations are reducible with the exception of the trivial $R=1$ case. The internal color labels $r, s$ are inert. We may insist only on the singlet representation of the unitary color group.

It turns out, however, that for finite $N$, the Green ansatz does not give all the realizations of $C_{N}$. In addition to the discrete family of representations obtained by the Green ansatz, there is a class of continuous representations which cannot be so obtained. This is most clearly seen for $N=1$ where the Lie algebra is that of $\mathrm{O}(2,1)$. The representations of $O(2,1)$ contain the discrete series of representations and a continuous set of representations. However, if $N \rightarrow \infty$ and we still have a Fock 
realization of the oscillators, Messiah and Greenberg have shown that only the Green ansatz obtains.

Let us now raise the question of cluster decomposition. The only irreducible representation of $\mathrm{O}(2 N+1)$ that remains irreducible when it is broken up into clusters is the fundamental spinor representation apart from the trivial representation. So any generalization of the Fermi anticommutation relations are reducible. The Green ansatz furnishes such a realization. Unfortunately it is not the most general one with such a cluster property. This problem was studied by J.L. Richards and E.C.G. Sudarshan, who demanded that entropy be an extensive quantity [16].

Another problem with parastatistics is that in a field theory the vacuum may not be cyclic. There are states not connected with the vacuum by a finite number of applications of the field. It is not known if the requirement of color singlet states cures this problem.

In concluding this section we restate the result, the irreducible realizations of the Green commutation relations are not equivalent to color. Conversely the Green ansatz which realizes color is not irreducible.

If the modes corresponding to $a_{j}, a_{j}^{\dagger}$ are for a spinning particle, then rotation and translation invariance in space puts further restrictions. By invariant integration with respect to the indices $j, k$ in the commutator

$$
\left[a_{j} a_{k}+a_{k} a_{j}, a_{l}^{\dagger}\right]=2\left(\delta_{j l} a_{k}+\delta_{k l} a_{j}\right),
$$

we obtain

$$
B_{j k}\left[a_{j} a_{k}+a_{k} a_{j}, a_{1}^{\dagger}\right]=B_{j k} a_{k}+B_{j l} a_{j} .
$$

If the invariant 'scalar' matrix $B_{j k}$ is symmetric, there is no problem, but for $B_{j k}$ antisymmetric the left side vanishes and the right side does not vanish leading to a contradictions. So para-Bose commutation relations are not consistent with halfinteger spin. Similarly, one can show that para-Fermi commutation relations are consistent for tensor fields.

\section{SPIN-STATISTICS IN LAGRANGIAN FIELD THEORY}

Introduce a Lagrangian density for a multi-component field $\varphi_{r}$ linear in the time derivative:

$$
L=\dot{\phi}_{r} A_{r s} \phi_{s}+m \phi_{r} B_{r s} \phi_{s}+L_{\text {int }},
$$

where the repeated indices are summed over. Since the Lagrangian density is invariant with respect to rotations, we can conclude that the matrices $A_{r s}$ and $B_{r s}$ have definite symmetry properties [17]. $B_{r s}$ is symmetric for tensor fields and antisymmetric for spinor fields. Due to the presence of the time derivative, $A_{r s}$ has the opposite symmetry; it is antisymmetric for tensor fields and symmetric for spinor fields. 
For this Lagrangian theory with the action functional

$$
A=\int d t \int d^{3} x L(\vec{x}, t),
$$

the Weiss-Schwinger action principle then asserts

$$
\begin{gathered}
i \delta \phi_{r}(\vec{x}, t)=\left[\phi_{r}(\vec{x}, t), \delta A\right] \\
\delta A=\dot{\phi}_{r} A_{r s} \delta \phi_{s} .
\end{gathered}
$$

Depending on whether the variation of the field $\delta \phi_{s}$ commutes or anticommutes with the field, we get the commutation relations

$$
\left[\varphi_{r}(\vec{x}, t), \varphi_{s}(\vec{y}, t) A_{s r}\right]=\delta_{r s} \delta(\vec{x}-\vec{y})
$$

or

$$
\left[\varphi_{r}(\vec{x}, t), \varphi_{r}(\vec{y}, t)\right]_{ \pm}=\left(A^{-1}\right)_{r s} \delta(\vec{x}-\vec{y}) .
$$

For commutation relations, $A$ and hence $A^{-1}$ must be antisymmetric, which is true for tensor fields and only for them. For anticommutation relation, $A$ and hence $A^{-1}$ must be symmetric, which is true for spinor fields and only for spinor fields.

The Action Principle and Newtonian group invariance of the action lead to the assertion that Bose quantization using commutators is only for tensor fields while Fermi quantization using anticommutators is only for spinor fields. To the extent that we have a particle interpretation, integer spin particles are bosons and half-integer spin particles are fermions. No use has been made of Lorentz invariance. The conclusion is therefore valid for ideal fluid hydrodynamics, which is Galilean invariant, and for phonons.

The red herring of antisymmetry in the internal symmetry labels providing the wrong spin-statistics connection is inadmissible for reasons given earlier in connection with Poincaré invariant theories.

For the spinor fields, the doubling of the number of components to deal with hermitian fields is seen as necessary also for providing the required number of canonical field components.

But if we try to quantize a scalar field canonically using anticommutators, we get an inconsistent theory. If the anticommutators satisfy

$$
\begin{aligned}
& \{\phi(\vec{x}, t), \phi(\vec{y}, t)\}_{+}=0 \\
& \{\phi(\vec{x}, t), \pi(\vec{y}, t)\}_{+}=i \delta(\vec{x}-\vec{y}) \\
& \{\pi(\vec{x}, t), \pi(\vec{y}, t)\}_{+}=0 .
\end{aligned}
$$

the theory is inconsistent; the vector space on which $\phi(\vec{x}, t)$ operates cannot have a positive-definite metric.

This is not avoided by making $\phi(\vec{x}, t)$ nonhermitian or a spinor field. For a nonhermitian scalar field with the anticommutators 


$$
\begin{aligned}
& \{\phi(\vec{x}, t), \phi(\vec{y}, t)\}_{+}=\left\{\phi(\vec{x}, t), \phi^{\dagger}(\vec{y}, t)\right\}_{+}=0 \\
& \{\pi(\vec{x}, t), \pi(\vec{y}, t)\}_{+}=\left\{\pi(\vec{x}, t), \pi^{\dagger}(\vec{y}, t)\right\}_{+}=0 \\
& \left\{\phi(\vec{x}, t), \pi^{\dagger}(\vec{y}, t)\right\}_{+}=i \delta(\vec{x}-\vec{y})=\left\{\phi^{\dagger}(\vec{x}, t), \pi(\vec{y}, t)\right\}_{+},
\end{aligned}
$$

the same inconsistency obtains. So, for a nonhermitian spinor field obeying such anticommutation relations, the theory is also inconsistent.

Pauli's proof, as mentioned above applies to free fields with a vacuum, for which the states of the fields are spanned by suitable symmetrized states of particles. In such a field theory the proof of the commutation relations is applicable immediately to particles. Pauli's proof may thus be thought of as applying to particles or to fields.

However, Lagrangian quantum field theory leads to infinities in direct calculation. While in many of these cases we can circumvent the infinities by renormalization, the renormalized field does not obey finite commutation relations. So for interacting fields we have either field commutation relations with unrenormalized fields or for noninteracting fields. In quantum theory a quantized field is equivalent to assemblies of identical particles. The statistics of the particles (that is the symmetry or antisymmetry or any other behavior) can be coded into the commutation properties of the field. If $\phi(x)$ is a field defined at time $t$, the Bose statistics of the quanta of the field depend on whether the canonical commutation relations are obeyed by the field and its conjugate. If $\phi(\vec{x}, t)$ contains both creation and destruction operators in the form

$$
\phi(\vec{x}, t)=\sum \frac{1}{\sqrt{2 \omega}}\left(a_{\vec{k}} e^{i \vec{k} \cdot \vec{x}-i \omega t}+a_{\vec{k}}^{\dagger} e^{i \vec{k} \cdot \vec{x}+i \omega t}\right),
$$

the canonical conjugate is its time derivative

$$
\pi(\vec{x}, t)=\dot{\phi}(\vec{x}, t)=\sum \frac{1}{\sqrt{2 \omega}}\left(-i \omega a_{\vec{k}} e^{i \vec{k} \cdot \vec{x}-i \omega t}+i \omega a_{\vec{k}}^{\dagger} e^{i \vec{k} \cdot \vec{x}+i \omega t}\right),
$$

and they obey the canonical relation

$$
[\phi(\vec{x}, t), \pi(\vec{x}, t)]=\sum_{k, j}\left[a_{\vec{k}}, a_{\vec{j}}^{\dagger}\right] e^{i \vec{k} \cdot(\vec{x}-\vec{y})}=\sum e^{i \vec{k} \cdot(\vec{x}-\vec{y})}=\delta(\vec{x}-\vec{y}) .
$$

But what if we had two fields

$$
\begin{aligned}
& \psi(\vec{x}, t)=\sum a_{\vec{k}} e^{i \vec{k} \cdot \vec{x}-i \omega t} \\
& \psi(\vec{x}, t)=\sum a_{\vec{k}}^{\dagger} e^{i \vec{k} \cdot \vec{x}+i \omega t} \quad ?
\end{aligned}
$$

Then we may choose either commutators or anticommutators to have the canonical forms

$$
\psi(\vec{x}, t) \psi^{\dagger}(\vec{x}, t) \pm \psi^{\dagger}(\vec{x}, t) \psi(\vec{x}, t)=\delta(\vec{x}-\vec{y}) .
$$

For example if $\psi, \psi^{\dagger}$ are both scalar fields, or $\psi, \psi^{\dagger}$ are both spinor fields, either commutators or anticommutators can be used to quantize the field. 


\section{TCP THEOREM IN QUANTUM FIELD THEORY WITHOUT LORENTZ INVARIANCE}

Returning to the three-dimensional space, not only can we provide the spin statistics theorem but also the TCP theorem [18]. We outline the arguments for spin0 and spin- $1 / 2$ cases.

For spin-0, we have a scalar field $\phi$ and its conjugate $\pi$. We may define the transformations of $\phi$ and $\pi$ under parity $\mathrm{P}$, charge conjugation $\mathrm{C}$, and weak time reversal $\mathrm{T}$ as follows

$$
\begin{aligned}
\phi(\vec{x}, t) & \rightarrow_{\mathrm{P}} \eta_{\mathrm{P}} \phi(-\vec{x}, t) \\
& \rightarrow_{\mathrm{C}} \eta_{\mathrm{C}} \phi(-\vec{x}, t) \\
& \rightarrow_{\mathrm{T}} \eta_{\mathrm{P}} \eta_{\mathrm{C}} \phi(\vec{x},-t) \\
\pi(\vec{x}, t) & \rightarrow_{\mathrm{P}} \eta_{\mathrm{p}} \pi(-\vec{x}, t) \\
& \rightarrow_{\mathrm{C}} \eta_{\mathrm{C}} \pi(\vec{x}, t) \\
& \rightarrow_{\mathrm{T}} \eta_{\mathrm{P}} \eta_{\mathrm{C}} \pi(\vec{x},-t)
\end{aligned}
$$

Hence under TCP, we have

$$
\begin{aligned}
& \phi(\vec{x}, t) \rightarrow \phi(-\vec{x},-t) \\
& \pi(\vec{x}, t) \rightarrow \pi(-\vec{x},-t) .
\end{aligned}
$$

Then the kinematic term in the Lagrangian is unaltered. The (nonderivative) interaction terms also retain their form and the action is unchanged.

For spin- $-1 / 2$ fields the kinematic terms are such that they yield the real equation of motion

$$
\psi_{r}=\left\{(\vec{\alpha} \cdot \vec{\nabla})_{r s}+B_{r s}\right\} \psi_{s} .
$$

This results from the Lagrangian

$$
L_{\min }=\psi_{r} \delta_{r s} \psi_{s}+\vec{\nabla} \cdot \psi_{r} \vec{\alpha}_{r s} \psi_{s}+\psi_{r} B_{r s} \psi_{s}
$$

The discrete transformations are

$$
\begin{aligned}
\psi_{r}(\vec{x}, t) & \rightarrow_{\mathrm{P}} \eta_{\mathrm{P}}(i \beta)_{r s} \psi_{s}(-\vec{x}, t) \\
& \rightarrow_{\mathrm{C}} \eta_{\mathrm{C}}\left(i \beta \gamma_{5}\right)_{r s} \psi_{s}(\vec{x}, t) \\
& \rightarrow_{\mathrm{T}} \eta_{\mathrm{C}} \eta_{\mathrm{P}}\left(i \beta \gamma_{5}\right)_{r s} \psi_{s}(\vec{x},-t)
\end{aligned}
$$

The case of two space dimensions needs to be addressed separately. Except for spin0 , all other fields with spin have two hermitian components. This is most clearly seen for two-dimensional vectors $\vec{A}$ and $\vec{B}$. Then both $\vec{A} \cdot \vec{B}$ and $\vec{A} \times \vec{B}$ are invariants, the first being symmetric and the second antisymmetric in $\vec{A}$ and $\vec{B}$. The generic kinematic Lagrangian density contains a suitable linear combination of the two invariants

$$
\begin{aligned}
\dot{\phi}_{r} A_{r s} \phi_{s} & \sim \cos \theta\left(\phi_{r} \dot{\phi}_{s}\right)+\sin \theta\left(\dot{\phi}_{r}^{*} \dot{\phi}_{s}\right) \\
A_{r s} & =\cos \theta \delta_{r s}+\sin \theta i \varepsilon_{r s}
\end{aligned}
$$


The factor of $i$ is necessary to assure the hermiticity of $A$. This angle $\theta$ is related to the anyon angle $\theta$ which defines the commutation property

$$
\phi_{r}(x) \phi_{s}(y)=e^{i \theta} \phi_{s}(y) \phi_{r}(x) .
$$

This is true for other spins including spin-1/2. So every possibility in the onedimensional representations of the (abelianized) braid group $B_{N}\left(\mathrm{R}^{2}\right)$ may be realized in field theory.

Consider a Newtonian quantum field, Poincaré covariant fields as well as Galilean covariant fields. But its scope is wider. We want to incorporate the fundamental Kirchhoff's law that emissivity and absorptivity are proportional. We implement this fundamental requirement by insisting that both kinematics and dynamics are expressed in terms of fields that contain creation and annihilation operators together as the positive and negative frequency parts of a single field. One easy way to bring this about is to use only hermitian fields in the formalism. A nonhermitian field can be so transcribed using two hermitian fields.

The equations of motion of tensor fields can be chosen real with the rotation group acting on the field components by real matrices. For spinor fields we need to double the components since the irreducible spinor in three dimensions is intrinsically complex. The usual Pauli matrices $\sigma_{1}, \sigma_{2}, \sigma_{3}$, have only $\sigma_{2}$ pure imaginary and the corresponding rotations purely real. When we double the components we may define an independent set $\tau_{1}, \tau_{2}, \tau_{3}$ of Pauli matrices and choose:

$$
\begin{aligned}
& \sigma_{1} \rightarrow \sigma_{1} \otimes \tau_{2} \\
& \sigma_{2} \rightarrow \sigma_{2} \otimes 1 \\
& \sigma_{3} \rightarrow \sigma_{3} \otimes \tau_{2} .
\end{aligned}
$$

Along with this we can define a set of vector matrices

and a scalar matrix

$$
\begin{aligned}
& \alpha_{1} \rightarrow \sigma_{3} \otimes \tau_{3} \\
& \alpha_{2} \rightarrow 1 \otimes \tau_{1} \\
& \alpha_{3} \rightarrow \sigma_{1} \otimes \tau_{2}
\end{aligned}
$$

$$
i \beta \rightarrow i 1 \otimes \tau_{2},
$$

so that we may consider

$$
\psi(\vec{x}, t) \rightarrow i \gamma_{5} \psi(-\vec{x},-t)
$$

To sum up,

$$
\begin{aligned}
& \alpha_{1}=\sigma_{3} \otimes \tau_{3} \\
& \alpha_{2}=1 \otimes \tau_{1} \\
& \alpha_{3}=\sigma_{1} \otimes \tau_{2} \\
& \beta=1 \otimes \tau_{2} \\
& \gamma_{5}=\sigma_{2} \otimes \tau_{1}
\end{aligned}
$$


These transformations are real and preserve the kinematic term. Since all dynamical terms must involve the spinor fields bilinearly, the action is invariant under this combined transformation. The action is thus TCP invariant; and hence the dynamics. Again we stress that relativistic invariance has not be used in the derivation.

\section{SUMMARY}

In summary we note that the usual Pauli derivation and the Burgoyne derivation are negative results, and it is not clear to what extent they apply to interacting particles. The same applies to the $\mathrm{C}^{*}$ algebraic results. This situation is not satisfactory and needs be improved. The study of the homotopy of the configuration spaces of identical particle showed what possible statistics may apply. But no deduction can be made by showing a continuous loop in the configuration space. The wave function may return to its original assigned values or to any other according to the representation of the homotopy group that applies. If we assume that only the trivial realization of the homotopy group is realized, we can "deduce" the spinstatistics connection. This would be a natural assumption but nevertheless an assumption.

To go beyond these arguments and to derive a positive result asserting the connection between spin and statistics, we recognize that the symmetry of the fundamental bilinear forms of two tensors or two spinors are respectively symmetric and antisymmetric. This basic property, together with the use of hermitian fields exclusively, allows us to deduce the spin-statistics connection. When the number of space dimensions is reduced to two, we have a generic bilinear form neither symmetric nor antisymmetric and parameterized by an angle [19].

The statement about the symmetry of the fundamental invariant bilinear forms does not hold in space of arbitrarily high dimension. The tensor invariant continues to be a bilinear symmetric form. But for spinors, the form may also be symmetric. In such a case even spinors must be quantized with Bose statistics [20]. Existence of internal symmetries cannot alter the spin-statistics relation.

We have also shown that in a local Newtonian quantum field theory the TCP theorem is automatically valid.

\section{REFERENCES}

1. Bacry, H., Am. J. Phys. 63 , 297-298 (1995).

2. Berry, M.V., and Robbins, J.M, Proc. Roy. Soc. A453, 1771-1790 (1997).

3. Imbo, T.D., and Sudarshan, E.C.G., Phys. Rev. Lett. 60, $481-483$ (1988); Imbo, T.D., Imbo, C.S., and Sudarshan, E.C.G., Ann. Inst. Henri. Poincaré 49, 387-396 (1988). 
4. Streater, G.F., and Wightman, A.S., PCT, Spin, Statistics and All That, W. A. Benjamin, New York, 1964.

5. Pauli, W., Progr. Theor. Phys. 5, 526-543 (1950).

6. Polyakov, M.A., Lett. Mod. Phys. A 3, 325 (1998)

7. Wightman, A.S., Phys. Rev. 101, 860-865 (1956).

8. Sudarshan, E. C. G., J. Math. Phys. 4, 1029-1036(1963).

9. Burgoyne, N., Nuovo Cimento 8, 607-609 (1958).

10. Haag, R., and Kastler, D., J. Math. Phys. 5, 848-861 (1964).

11. Wigner, E.P., Phys. Rev. 77, 711 (1950).

12. Ryan, C., and Sudarshan, E.C.G., Nucl. Phys. 47, 207-211 (1963).

13. Bhabha, H.J., Rev. Mod. Phys. 21, 451 (1949).

14. Kamefuchi, S., and Takahashi, Y., Nucl. Phys. 36, 177-206 (1962).

15. Murnaghan, F.D, Theory of Group Representations, Johns Hopkins Press, Baltimore, 1938.

16. Richard, J.L., and Sudarshan, E.C.G, J. Math. Phys. 14, 1170-1175 (1973).

17. Duck, I.M., and Sudarshan, E.C.G., Am. J. Phys. 66, 284-303 (1998).

18. Luders, G., Z. f. Physik 133, 325-330 (1954).

19. Wilczek, F, Int. J. Mod. Phys. A 3, 2827-2853 (1998).

20. Boya, L.J., and Sudarshan, E.C.G., unpublished. 